EPJ manuscript No.

(will be inserted by the editor)

\title{
Space-time evolution of hadronization
}

\author{
Alberto Accardi ${ }^{1}$ a \\ Dept. of Physics and Astronomy, Iowa State U., Ames, IA 50011, USA
}

\begin{abstract}
Beside its intrinsic interest for the insights it can give into color confinement, knowledge of the space-time evolution of hadronization is very important for correctly interpreting jet-quenching data in heavy ion collisions and extracting the properties of the produced medium. On the experimental side, the cleanest environment to study the space-time evolution of hadronization is semi-inclusive Deeply Inelastic Scattering on nuclear targets. On the theoretical side, 2 frameworks are presently competing to explain the observed attenuation of hadron production: quark energy loss (with hadron formation outside the nucleus) and nuclear absorption (with hadronization starting inside the nucleus). I discuss recent observables and ideas which will help to distinguish these 2 mechanisms and to measure the time scales of the hadronization process.
\end{abstract}

PACS. 25.30.-c - 25.75.-q - 24.85.+p - 13.87.Fh

\section{Introduction}

One of the most striking experimental discoveries in the heavy-ion program at the Relativistic Heavy Ion Collider (RHIC) has been the the suppression of large transverse momentum hadron production in nucleus-nucleus $(\mathrm{A}+\mathrm{A})$ collisions compared to proton-proton $(\mathrm{p}+\mathrm{p})$ collisions 1 . The observable of interest is the ratio of the hadron transverse momentum $\left(p_{T}\right)$ spectrum in $\mathrm{A}+\mathrm{B}$ collision in a given centrality class (c.cl.), normalized to binary scaled $\mathrm{p}+\mathrm{p}$ collisions by the inverse thickness function $T_{A B}$ and finally divided by the $\mathrm{p}+\mathrm{p}$ spectrum:

$$
R_{A B}=\frac{1}{T_{A B}(\text { c.cl. })} \frac{d N^{A+B \rightarrow h+X}}{d p_{T}^{2} d y}(\text { c.cl. }) / \frac{d \sigma^{p+p \rightarrow h+X}}{d p_{T}^{2} d y}
$$

In the absence of nuclear effects, one would expect $R_{A B}=$ 1. By comparing the measured $R_{A u A u} \approx 0.2$ in $\mathrm{Au}+\mathrm{Au}$ collisions to the mild deformation of $p_{T}$ spectra observed in deuteron-gold $(\mathrm{d}+\mathrm{Au})$ collisions, one concludes that the large suppression of $R_{A u A u}$ is due to the hot and dense medium produced in $\mathrm{Au}+\mathrm{Au}$ collision, also called "hot nuclear matter", see Fig. 1] This measurement is one of the keys to the claimed discovery of the Quark-Gluon Plasma (QGP) at RHIC [2].

The suppression of hadron production in $\mathrm{A}+\mathrm{A}$ collisions, has been successfully described in terms of parton energy loss due to medium-induced gluon bremsstrahlung, allowing so-called "jet tomography" studies of the QGP 3.4. However, this success has been recently questioned. Gluon radiation off heavy quarks is expected on theoretical grounds to be suppressed at small angles compared

\footnotetext{
a Based on a talk given at "Hot Quarks 2006", Villa Simius, Sardinia, Italy, May 15-20, 2006
}

to light quarks, implying a smaller suppression for $D$ and $B$ mesons than for $\pi$ mesons [5]. However, the measured suppression of single non-photonic electrons at RHIC [6], which are the decay product of $B$ and $D$ mesons, is of similar magnitude for pions contrary to theoretical expectations 7. The common assumption of neglecting elastic parton energy loss in $R_{A A}$ computations has been recently reexamined [8, but the effect seems insufficient to explain the data, at least within conventional schemes for treating the running coupling constant 9 . As further assumption that needs to be tested and will be addressed in this paper, is that the quark which fragments into the observed hadron traverses the whole medium and hadronizes well outside it. If untrue, in-medium interactions and screening of the hadronizing system would need to be accounted for.

Hadron suppression has also been observed in fixed target Deeply Inelastic lepton-nucleus Scattering (nDIS). In this case, the medium which induces the attenuation is the target nucleus itself, also called "cold nuclear matter", see Fig 1] Experimental data are usually presented in terms of the "multiplicity ratio" $10,11,12,13,14$

$$
R_{M}^{h}\left(z_{h}\right)=\frac{1}{N_{A}^{D I S}} \frac{d N_{A}^{h}\left(z_{h}\right)}{d z_{h}} / \frac{1}{N_{D}^{D I S}} \frac{d N_{D}^{h}\left(z_{h}\right)}{d z_{h}},
$$

i.e., the single hadron multiplicity on a target of mass number $A$ normalized to the multiplicity on a deuteron target as a function of the hadron's fractional energy $z_{h}=$ $E_{h} / \nu$, where $\nu$ is the virtual photon energy. The ratios in the numerator and denominator cancel to a large extent initial state effects like the modifications of parton distribution functions due to shadowing and EMC effects, exposing the nuclear modifications of the fragmentation process: if we assume factorization formulae to be valid, 


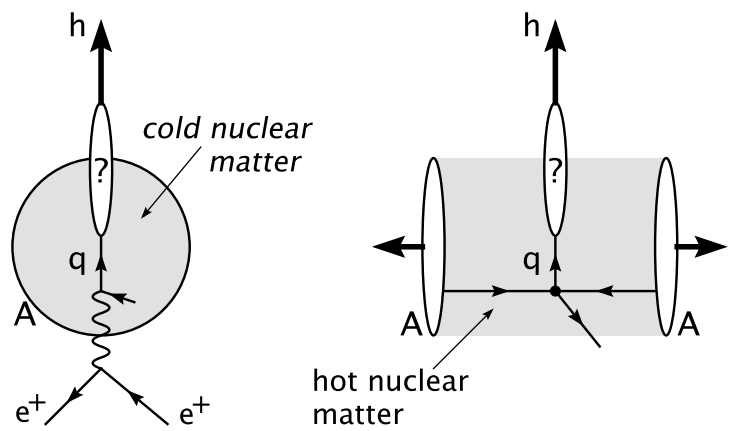

Figure 1. Left: Lepton-nucleus scattering. The hadronizing quark travels through the target nucleus. Right: Nucleusnucleus scattering. The hadronizing parton travels through the medium produced in the collisions.

we have at leading order $R_{M} \approx D_{h}^{A} / D_{h}^{D}$, i.e., the ratio of the fragmentation functions (FF) in the nucleus $\mathrm{A}$ and in deuteron. If no nuclear effects modify the fragmentation process we would expect $R_{M} \approx 1$. In fact, what is experimentally observed is hadron suppression in the $z_{h}=0.2-1$ and $\nu=2-20 \mathrm{GeV}$ range at HERMES 11, 12, 13, and in the $\nu=20-200 \mathrm{GeV}$ at the EMC experiment [10]. The flavor dependence of the multiplicity ratio has also been measured [12]13, showing suppression for pions, kaons and antiprotons. Protons are enhanced at $z_{h} \lesssim 0.4$ and suppressed above: this is a "proton anomaly" analogous to the "baryon anomaly" observed in $\mathrm{p}+\mathrm{A}$ and $\mathrm{A}+\mathrm{A}$ collisions [1] 15]. Both the quenching and the enhancement increase with $A$. Data binned in $\nu$, and in the photon virtuality $Q^{2}$ are also available from HERMES. Very high-statistics measurements will be available in the near future from the CLAS experiment at Jefferson Labs 14], with some preliminary results already presented [16].

The cleanest environment to address nuclear modifications of hadron production is nuclear DIS: it allows to experimentally control many kinematic variables; the nuclear medium (i.e., the nucleus itself) is well known; the multiplicity in the final state is low, allowing for precise measurements. Moreover, the nucleons act as femtometerscale detectors of the hadronizing quark, allowing to experimentally study its space-time evolution into the observed hadron. Hadron suppression at HERMES and CLAS is of direct relevance to RHIC physics. In both cases the hadronizing quark has to traverse a length of (hot or cold) nuclear matter of the size of a nuclear radius, see Fig 1 Moreover, in the HERMES experiment $\nu \approx 2-25 \mathrm{GeV}$ and $z_{h} \approx 0.2-1$, so that hadrons are observed over an energy range $E_{h}=z_{h} \nu=2-20 \mathrm{GeV}$. (measurements at CLAS with $E_{\text {beam }}=5,11 \mathrm{GeV}$ will likewise explore the $E_{h}=2-10 \mathrm{GeV}$ range). For midrapidity hadron production in A+A collisions at RHIC $E_{h} \approx p_{T} \approx 2-20 \mathrm{GeV}$, roughly equal to the hadron energy range at HERMES.

Information about parton propagation in cold nuclear matter is needed as an input for the interpretation of data in $\mathrm{A}+\mathrm{A}$ collisions. In this case one wants to use hadron suppression as a tool to extract the properties of the hot QGP created in the collision. To this purpose we need to develop well calibrated computational tools to relate the magnitude of hadron suppression to properties of the QGP like its density and temperature. Assuming long lived partons, hadron suppression at RHIC would be attributed to parton energy loss, leading to a medium temperature of $T \approx 400 \mathrm{MeV} 4$, in excess of the critical temperature $T_{c} \approx 170 \mathrm{MeV}$ for the QGP phase transition. If, on the contrary, hadronization started on the nuclear radius scale or before, in-medium interactions should also be accounted for, leading to a different, presumably lower $T$. A precise knowledge of parton propagation and hadronization mechanisms obtained from nDIS data is essential for testing and calibrating our theoretical tools, and to determine the properties of the QGP produced at RHIC.

\section{Formation time estimates}

The key quantity we need to investigate is the hadronization time scale. Since hadronization is a non perturbative process, one has to resort to phenomenological models to describe it 17 18, 19, 20, 21, 22, 23. However, a few features are expected on general grounds. Due to color confinement, the struck quark must neutralize its color at some stage, say by picking up an antiquark from the vacuum or the surrounding medium. I call this color neutral $q \bar{q}$ pair a "prehadron" $h_{*}$, and the time for its formation the "prehadron formation time" $t_{*}$ (some authors prefer to call it the "production" time). This is a relevant time scale since gluon bremsstrahlung off the struck quark stops after color neutralization; moreover, the prehadron quickly develops a cross section of the order of the hadronic one, leading to its nuclear absorption. Subsequently, the prehadron wave function collapses on the observed hadron $h$ wave function, and the corresponding time is called "hadron formation time" $t_{h}$. A final caveat: it is difficult to rigorously define the concept of formation time in field theory, so that in the following discussions it is used as a working tool.

\subsection{Long formation time: energy loss models}

The average hadron formation time $\left\langle t_{h}\right\rangle$ can be considered as the time for the struck partons to build up its color field and to develop the hadronic wave function 24. In the hadron rest frame this time is related to the hadron radius, and in the laboratory frame it is boosted to:

$$
\left\langle t_{h}\right\rangle \propto R_{h} \frac{E_{h}}{m_{h}}=R_{h} \frac{z \nu}{m_{h}}
$$

For a $10 \mathrm{GeV}$ pion at HERMES, we have $\left\langle t_{h}\right\rangle \approx 50 \mathrm{fm}$ $\gg R_{A}$. Note also that the scale for hadron formation is set by $\kappa_{h}=m_{h} / R_{h} \approx 0.2 \mathrm{GeV} / \mathrm{fm}$. This estimate is used in energy loss models [17,18, to assume that hadronization starts outside the medium with a decreased parton energy due to multiple parton scatterings and induced gluon bremsstrahlung. These models are fairly successful in describing $R_{M}$ at HERMES, see Fig. 2

More in detail, the Ref. [17] computes parton rescatterings and gluon radiation in pQCD including Feynman 

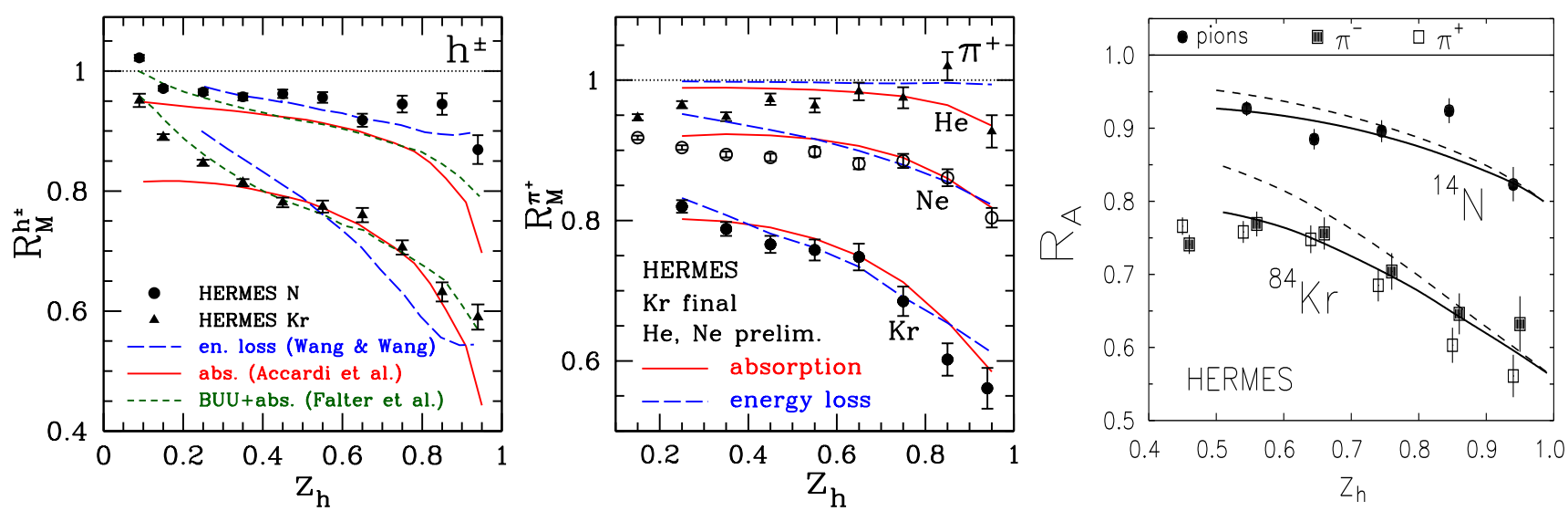

Figure 2. Left:Multiplicity ratio for $h^{ \pm}$at HERMES [12] compared to the energy loss model of Ref. [17 and the absorption models of Ref. 21 23. Center: pure absorption model [21 27] (solid) and energy loss model 27 18 (dashed). Right: Color dipole model from Ref. [22] (dashed: absorption only, solid: absorption and induced energy loss). Data are from Ref. [11 12 13].

diagrams up to twist-4. Fragmentation of both the struck quark and the radiated gluon is included. The modification of the FF depends on 1 parameter, the strength of parton-parton correlations in the nucleus. The modified FF so obtained can be modeled to a good accuracy by shifting $z_{h}$ in the leading-twist fragmentation function:

$$
\tilde{D}\left(z_{h}\right) \longrightarrow \frac{1}{1-\Delta z_{h}} D\left(\frac{z_{h}}{1-\Delta z_{h}}\right)
$$

where $\Delta z_{h}=\Delta E_{q} / \nu$ is the quark's fractional energy loss, and $\Delta E_{q} \approx 0.6\left\langle z_{g}\right\rangle$ with $\left\langle z_{g}\right\rangle$ the average fractional energy carried away by the radiated gluon.

In Ref. 18 the parton energy loss is treated in the BDMS formalism [25, which takes into account mediuminduced multiple soft gluon emission and fluctuations in the energy loss. Modified FF are computed as the average of the $z_{h}$-shifted FF in Eq. (4):

$$
\tilde{D}_{f}^{h}\left(z_{h}\right)=\int_{0}^{(1-z)} d \Delta z \mathcal{P}\left(\Delta z ; \hat{q}, L_{q}\right) \frac{1}{1-\Delta z} D_{f}^{h}\left(\frac{z_{h}}{1-\Delta z}\right) .
$$

The "quenching weight" $\mathcal{P}(\Delta z)$ is the probability distribution of a fractional energy loss $\Delta z=\Delta E_{q} / \nu$ [25]26], and $L_{q}$ the quark's in-medium path length. The medium is characterized by the transport coefficient $\hat{q}$, which measures the average momentum transfer per unit path-length from the medium to the parton. When also taking into account a realistic medium geometry and finite medium size corrections to the quenching weight, the model $27 \mathrm{can}$ well describe HERMES data, see Fig. 2 .

\subsection{Short formation time: nuclear absorption models}

A successful non perturbative model of hadronization is the Lund string model 28 . The confined color field stretching from the struck quark to the rest of the nucleus is modeled as a string of tension $\kappa_{\text {str }} \approx 1 \mathrm{GeV} / \mathrm{fm}$. Prehadron formation is identified with the $q \bar{q}$ pair production which breaks the string in smaller pieces 19 . Hadrons are formed when a quark and an antiquark at the endpoint of a string fragment meet. Average formation times can be analytically computed [19 20,21]:

$$
\begin{aligned}
& \left\langle t_{*}\right\rangle=f\left(z_{h}\right)\left(1-z_{h}\right) \frac{z_{h} \nu}{\kappa_{s t r}} \\
& \left\langle t_{h}\right\rangle=\left\langle t_{*}\right\rangle+\frac{z_{h} \nu}{\kappa_{s t r}} .
\end{aligned}
$$

The factor $z_{h} \nu$ can be understood as a Lorentz boost factor. The $\left(1-z_{h}\right)$ factor is due to energy conservation: a high- $z_{h}$ hadron carries away an energy $z_{h} \nu$; the string remainder has a small energy $\epsilon=\left(1-z_{h}\right) \nu$ and cannot stretch farther than $L=\epsilon / \kappa_{s t r}$. Thus the string breaking must occur on a time scale proportional to $1-z_{h}$. The function $f\left(z_{h}\right)$ is only a small deformation of $\left\langle t_{*}\right\rangle$. At HER$\operatorname{MES}\left\langle t_{*}\right\rangle \approx 4 \mathrm{fm}<R_{A}$ and $\left\langle t_{h}\right\rangle \approx 6-10 \mathrm{fm} \gtrsim R_{A}$. The hadron is typically formed at the periphery or outside the nucleus so that its interaction with the medium is negligible. However, the prehadron is formed well inside and can start interacting with the nucleus. The nuclear absorption model of Ref. 21] can successfully explain $R_{M}$ measurements at HERMES in terms of prehadron-nucleon inelastic scatterings with the above formation times estimate, see Fig. 2. The prehadron-nucleon inelastic cross-section is $\sigma_{*}(\nu)=0.35 \sigma_{h}(\nu)$ proportional to the experimental hadron-nucleon cross section $\sigma_{h}$. The proportionality factor is fitted to $\pi^{+}$production data on a Kr target 12. The prehadron survival probability $S_{*}$ is computed in terms of transport equations. Neglecting hadron absorption,

$$
S_{*}=\int d^{2} b d y \rho_{A}(b, y) \times \int_{y}^{\infty} d x \frac{e^{-\frac{x-y}{\left\langle t_{*}\right\rangle}}}{\left\langle t_{*}\right\rangle} e^{-\sigma_{*} \int_{x}^{\infty} d s A_{A}(b, s)}
$$

where $(b, y)$ is the $\gamma^{*}$-q interaction point, $\rho_{A}$ is the nuclear density, and one can recognize exponential probability distributions for prehadron and hadron formation.

In Ref. 22 the formation of a leading hadron $\left(z_{h} \gtrsim\right.$ $0.5)$ is described in a pQCD inspired model. The struck 

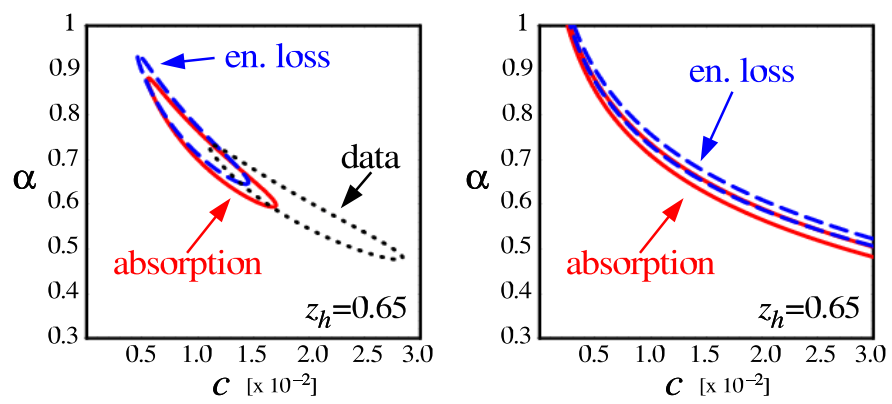

Figure 3. Left: results of the $R_{M}=c A^{\alpha}$ fit for $\{\mathrm{He}, \mathrm{N}, \mathrm{Ne}, \mathrm{Kr}\}$ at $\mathrm{z}=0.65$ (solid: absorption; dashed: energy loss; dotted: data 12]). Right: computations including only heavy nuclei $\{\mathrm{Kr}, \mathrm{Sn}, \mathrm{Xe}, \mathrm{W}, \mathrm{Au}, \mathrm{Pb}\}$.

quark radiates a gluon according to the Bethe-Heitler radiation spectrum. The gluon then splits into a $q \bar{q}$ pair, and the $\bar{q}$ recombines with the struck $q$ to form the leading prehadron. Medium interaction and evolution of the prehadron into the observed hadron is computed in a pathintegral formalism for dipole propagation. The prehadron formation time is identified with the time at which the gluon becomes decoherent with the struck quark. The probability distribution in the prehadron formation time can be computed, and the average $\left\langle t_{*}\right\rangle$ is

$$
\left\langle t_{*}\right\rangle \propto\left(1-z_{h}\right) \frac{z_{h} \nu}{Q^{2}} .
$$

The scale is set by $\kappa_{\text {dip }}=Q^{2} \approx 10 \mathrm{GeV} /$ fm at HERMES, and $\left\langle t_{*}\right\rangle \lesssim 5 \mathrm{fm}$ at $z_{h}>0.5$. This model can successfully describe leading hadron suppression, see Fig. 2 right.

In Ref. 23] a different space-time picture of hadronization is advocated. Prehadrons are formed at $t_{*}=0$, and hadrons are formed at $t_{h}=\left(E_{h} / m_{h}\right) \tau_{0}$ with $\tau_{0}=0.5 \mathrm{fm}$. The leading prehadron interacts with the medium with a reduced hadronic cross-section. Subleading prehadrons do not interact with the medium until hadron formation. This picture is then embedded in a Monte Carlo transport model. A good description of HERMES data can be achieved, see Fig. 2 .

\section{Can we distinguish energy loss from hadron absorption?}

Most of the difference in the time estimates quoted in the previous Section lies in the different scale $\kappa$ used. E.g., $\kappa_{h} \approx 0.2 \kappa_{\text {str }}$ leads to the rather large $\left\langle t_{h}\right\rangle \approx 50 \mathrm{fm}$ quoted in the energy loss model estimate instead of $\left\langle t_{h}\right\rangle \approx 10 \mathrm{fm}$ quoted in the Lund model estimate. In the second case there would be no justification for neglecting the interactions of the forming hadron field with the nucleus. As the choice of the scale $\kappa$ is a debatable and model-dependent matter 22 29, it is very important to look for observables which are able to distinguish energy loss models and absorption models, or to directly detect in-medium hadronization effects.

\subsection{Mass number dependence}

In first approximation, one expects $1-R_{M}^{h} \propto A^{2 / 3}$ in energy loss models because the average energy loss $\Delta E_{q} \propto$ $\left\langle L_{q}^{2}\right\rangle \propto A^{2 / 3}$, due to the Landau-Pomeranchuk-Migdal interference in QCD 33 . On the other hand, in absorption models the survival probability is proportional to the amount of traversed matter, so that $1-R_{M}^{h} \propto\left\langle L_{A}\right\rangle \propto$ $A^{1 / 3}$. Therefore a simple analysis of the $A$-dependence of $R_{M}^{h}$ should clearly signal which model is correct.

This argument fails for absorption models 21 30. If the prehadron were produced always at the $\gamma^{*}$-quark interaction point (i.e., $t_{*}=0$ ) then $R_{M}=c A^{1 / 3}$ at all orders in $A^{1 / 3}$. However, if we allow for a nonzero $\left\langle t_{*}\right\rangle$, its dimension must be neutralized by the nuclear radius $R_{A}$, introducing extra powers of $A^{1 / 3}$. Quite generally, if the probability distribution for the prehadron formation length is finite at zero formation length, then $R_{M}^{h} \propto A^{2 / 3}+O(A)$, the same power found in energy loss models.

Then, we can study the breaking of the $A^{2 / 3}$ law. To this purpose, it was proposed in [21] to select a set of targets $\left\{A_{1}, A_{2}, \ldots, A_{n}\right\}$, fix the $z$ bin, and perform a fit of the form $1-R_{M}^{h}(z)=c(z) A^{\alpha(z)}$ with $c$ and $\alpha$ free parameters. Results are presented in terms of $2 \sigma$ confidence contours in the $(c, \alpha)$ plane. As shown in Fig. 3left, energy loss 27] and absorption models [21,27] are indistinguishable. The same holds true for all $z$ bins. Experimental data are described by an $A^{\alpha}$ power law with $\alpha=0.61 \pm 0.14$, compatible with $\alpha=2 / 3$ but excluding $\alpha=1 / 3$. Increasing the number of targets and the span in atomic number does not help in separating the 2 models, either, but clearly shows a non negligible breaking of the $A^{2 / 3}$ law at $A \gtrsim 80$ [21 27], see Fig. 3] right.

\subsection{Formation time scaling}

In Ref. 31] I conjecture that $R_{M}$ should not depend on $z_{h}$ and $\nu$ separately but should depend on a combination of them:

$$
R_{M}=R_{M}\left[\tau\left(z_{h}, \nu\right)\right]
$$

where the scaling variable $\tau$ is defined as

$$
\tau=C z_{h}^{\lambda}\left(1-z_{h}\right) \nu
$$

The scaling exponent $\lambda$ is introduced as a way of approximating and summarizing the scaling behavior of experimental data and theoretical models. It can be obtained by a best fit analysis of data or theoretical computations. The proportionality constant $C$ cannot be determined by the fit. A possible scaling of $R_{M}$ with $Q^{2}$ is not considered in this analysis because of its model dependence, and because of the mild dependence of HERMES data on $Q^{2}$. As discussed below, the proposed functional form of $\tau$, Eq. (9), is flexible enough to encompass both absorption models and energy loss models. The 2 classes of models are distinguished by the value of the scaling exponent: a positive $\lambda \supsetneqq 0$ is characteristic of absorption models, while 

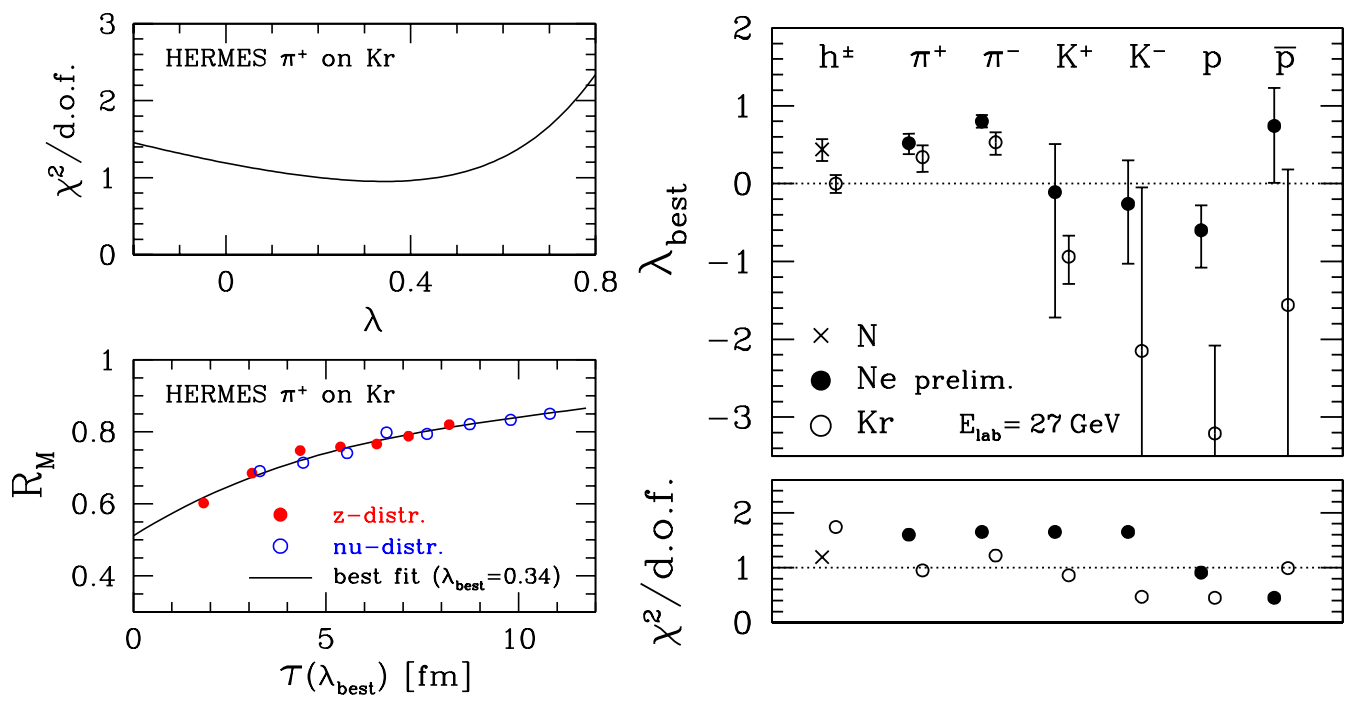

Figure 4. Left: An example of the fit procedure described in Section 3.2 applied to HERMES data for $\pi^{+}$production on a Kr target [12. Upper panel: $\chi^{2}$ as a function of $\lambda$. Lower panel: $R_{M}(\tau)$ with $\tau$ computed at $\lambda_{\text {best }}=0.34$. Right: The scaling exponent $\lambda_{\text {best }}$ extracted from HERMES data on charged and identified hadrons at $E_{\text {lab }}=27 \mathrm{GeV} 111213$ (only statistical errors included in the fit). Error bars correspond to 1 standard deviation. The bottom panel shows the $\chi^{2}$ per degree of freedom.

a negative $\lambda \lesssim 0$ is characteristic of energy loss models. Thus, the exponent $\lambda$ extracted from experimental data can identify in the experimental data the leading mechanism for hadron suppression in nDIS.

The scaling of $R_{M}$ is quite natural in the context of hadron absorption models 1920,2122 . Indeed, prehadron absorption depends on the in-medium prehadron path length, which depends solely on the prehadron formation time $\left\langle t_{*}\right\rangle$ as long as $\left\langle t_{*}\right\rangle \lesssim R_{A}$. As argued in Section 2. $\left\langle t_{*}\right\rangle \propto f\left(z_{h}\right)\left(1-z_{h}\right) z_{h} \nu$ which is well described by the proposed scaling variable $\tau$ with $\lambda>0$. E.g., in the Lund model $\lambda \approx 0.7$.

In energy loss models [17, 18,27] the scaling is less obvious. For the purpose of discussing the scaling properties of $R_{M}$, we can consider the model of Ref. 18,27 and neglect finite medium size corrections and finite quark energy corrections. If we further neglect energy loss fluctuations, we can approximate $R_{M} \approx \widetilde{D}_{A}\left(z_{h}\right) / D\left(z_{h}\right)$ and obtain

$$
R_{M} \approx \frac{1}{1-\langle\epsilon\rangle / \nu} D\left(\frac{z_{h}}{1-\langle\epsilon\rangle / \nu}\right)\left[D\left(z_{h}\right)\right]^{-1},
$$

where the average energy loss $\langle\epsilon\rangle=\int_{0}^{\left(1-z_{h}\right) \nu} d \epsilon \epsilon \mathcal{P}(\epsilon)$ $/ \int_{0}^{\left(1-z_{h}\right) \nu} d \epsilon \mathcal{P}(\epsilon)=f\left[\left(1-z_{h}\right) \nu\right]$ is a function of the energy $\left(1-z_{h}\right) \nu$ not carried away by the observed hadron. Next, we can approximate the $\mathrm{FF}$ using the parametrization of Ref. 32 at $Q^{2}=2 \mathrm{GeV}^{2}$ and obtain

$$
R_{M} \approx \frac{1}{\left(1-\frac{1}{\nu} f\left[\left(1-z_{h}\right) \nu\right]\right)^{\alpha+\beta+1}}\left(1-\frac{f\left[\left(1-z_{h}\right) \nu\right]}{\left(1-z_{h}\right) \nu}\right)^{\beta}
$$

This shows an approximate scaling with $\left(1-z_{h}\right) \nu$, which implies scaling of $R_{M}$ with respect to $\tau$ with $\lambda \approx 0$. A similar argument holds for the model of 17 . When performing the scaling analysis of the full models one finds in general $\lambda \lesssim 0$ 31].
The HERMES experiment measures $R_{M}$ binned in $z_{h}$ and integrated over $\nu$ and $Q^{2}$ (" $z_{h}$ distributions") or binned in $\nu$ and integrated over $z_{h}$ and $Q^{2}$ (" $\nu$ distributions"). Eq. (8) is fitted to the combined $z_{h^{-}}$and $\nu$ distributions, and the scaling exponent $\lambda$ is determined by $\chi^{2}$ minimization. An example of this procedure is illustrated in Fig. ??. Details can be found in Ref. 31.

The scaling exponents $\lambda_{\text {best }}$ extracted from HERMES data at $E_{l a b}=27 \mathrm{GeV}$ 11,12,13 for different hadron flavors produced on $\mathrm{N}, \mathrm{Ne}$ and $\mathrm{Kr}$ targets are shown in Fig. ??. In all cases $\chi^{2} /$ d.o.f. $\lesssim 1.6$, which proves that $R_{M}$ scales with $\tau$. The central result of this analysis is that pion data exhibit a clear $\lambda_{\text {best }} \approx 0.4 \supsetneqq 0$. As discussed, this shows the dominance of the prehadron absorption mechanism as opposed to the energy loss mechanism, or in other words is a signal of in-medium prehadron formation, with formation times $\left\langle t_{*}\right\rangle \lesssim R_{A}$.

\section{3 $p_{T}$ broadening}

The scaling analysis just described gives only indirect evidence for a short formation time, and cannot measure its absolute scale. An observable which is more directly related to the prehadron formation time is the hadron's transverse momentum broadening in DIS on a nuclear target compared to a proton or deuteron target 22. Indeed, when a hadron is observed in the final state, neither the quark nor the prehadron could have had inelastic scatterings. The prehadron-nucleon elastic cross section is very small compared to the quark cross section, so that the hadron's $p_{T}$-broadening originates dominantly during parton propagation. As shown in 33 34, the quark's momentum broadening $\Delta p_{T}^{2}$ is proportional to the quark pathlength in the nucleus. If the prehadron formation time has 
the form (9) as argued in the last section, we obtain:

$$
\Delta p_{T}^{2} \propto\left\langle t_{*}\right\rangle \propto z_{h}^{\lambda}\left(1-z_{h}\right) \nu,
$$

unless the distance of the quark production point from the surface of the nucleus is smaller than $\left\langle t_{*}\right\rangle$. Then we should expect a decrease of $\Delta p_{T}^{2}$ with increasing $z_{h}$. This would be a clear and model-independent signal of in-medium prehadron formation: indeed, if the quark were traveling through the whole nucleus before prehadron formation $\Delta p_{T}^{2}$ would only depend on the nucleus size and not on $z_{h}$. A related observable is the $z_{h}$-dependence of the Cronin effect, which is likewise expected to decrease with increasing $z_{h}$ [22].

The CLAS collaboration can perform multi-differential $p_{T}$-broadening measurements in all kinematic variables thanks to a very high beam luminosity. A few preliminary data from CLAS are already available [16. The HERMES collaboration is also studying the $p_{T}$-broadening at a larger beam energy but with a lower statistics. The scaling analysis proposed in the previous section will be useful to cross-check the results of these measurements.

\section{Conclusions and perspectives}

Use of hard processes to probe medium processes in $\mathrm{A}+\mathrm{A}$ collisions requires a detailed understanding of the hadronization process, which can be studied in lepton-nucleus scatterings. The scaling analysis 31 of pion attenuation at HERMES demonstrates for the first time a scaling of the hadron attenuation ratio $R_{M}$ which is compatible with a short prehadron formation time of the order or smaller than the nuclear radius. Thus, it favors prehadron absorption as dominant mechanism for hadron suppression instead of gluon radiation off a struck quark. This conclusion will be soon checked by hadron $p_{T}$-broadening data.

Much more can be studied in lepton-nucleus scatterings. (i) In the meson sector, the suppression of $\eta$ mesons at RHIC, which is of similar magnitude than for $\pi$, seems to favor long-lived partons 35. Measuring $\eta$ and heavier meson attenuation at HERMES and CLAS will check the correctness of such interpretation. (ii) Understanding the proton anomaly in nDIS will shed light on baryon transport in nuclear matter and on the baryon anomaly observed in $\mathrm{p}+\mathrm{A}$ and $\mathrm{A}+\mathrm{A}$ collisions. Measurements of $\Lambda$ and other baryons at HERMES and CLAS will be needed in this respect. (iii) Neither HERMES nor CLAS are able to study the hadronization of heavy quarks, because of limited luminosity and limited Bjorken's $x$ coverage, respectively. The proposed Electron-Ion Collider [36] is well suited for such studies, thanks to its low- $x$ coverage and high luminosity. Study of D and B meson suppression will settle the single electron puzzle at RHIC and will put interpretation of LHC data on a firmer ground. Study of "normal" $J / \psi$ suppression will help in distinguishing competing mechanism and in building a precise baseline for measurements of the "anomalous" suppression in A+A collisions.
I am very grateful to the organizers of this workshop for the financial support they offered me. I would like to thank V. Muccifora, D. Grünewald, H. J. Pirner for their collaboration on many results discussed in this work, and J. W. Qiu, P. Di Nezza, F. Arleo, C. Salgado, T. Falter, K. Gallmeister for valuable discussions. Figure 1 has been realized using JaxoDraw by D. Binosi and L. Theussl. This work is partially funded by the US Department of Energy grant DE-FG02-87ER40371.

\section{References}

1. I. Arsene et al. [BRAHMS], Nucl. Phys. A 757, 1 (2005); B. B. Back et al. [PHOBOS], Nucl. Phys. A 757, 28 (2005); J. Adams et al. [STAR], Nucl. Phys. A 757, 102 (2005); K. Adcox et al. [PHENIX], Nucl. Phys. A 757 (2005) 184.

2. Brookhaven National Laboratory announcement, April 2005, http://www.bnl.gov/bnlweb/pubaf/pr/PR_ display.asp?prID $=05-38$.

3. M. Gyulassy, I. Vitev, X. N. Wang and B. W. Zhang, in "Quark Gluon Plasma 3", R.C. Hwa and X.N. Wang eds., World Scientific, Singapore arXiv:nucl-th/0302077.

4. I. Vitev, J. Phys. G 30 (2004) S791.

5. Y. L. Dokshitzer and D. E. Kharzeev, Phys. Lett. B 519 (2001) 199

6. J. Bielcik [STAR], arXiv:nucl-ex/0511005 and these proceedings; S. S. Adler et al. [PHENIX], Phys. Rev. Lett. 96 (2006) 032301.

7. M. Djordjevic et al., Phys. Lett. B 632 (2006) 81; N. Armesto et al., Phys. Lett. B 637 (2006) 362

8. S. Wicks et al., arXiv:nucl-th/0512076

9. A. Peshier, arXiv:hep-ph/0605294 and these proceedings arXiv:hep-ph/0607275.

10. J. Ashman et al. [EMC], Z. Phys. C 52 (1991) 1.

11. A. Airapetian et al. [HERMES Collaboration], Eur. Phys. J. C 20 (2001) 479

12. A. Airapetian et al. [HERMES], Phys. Lett. B 577 (2003) 37 ;

13. G. Elbakian et al [HERMES], Proceedings "DIS 2003", St.Petersburg, April 23-27, 2003; V.T. Kim and L.N. Lipatov eds., page 597.

14. B. A. Mecking et al. [CLAS], Nucl. Instrum. Meth. A 503 (2003) 513; W. K. Brooks, FizikaB 13 (2004) 321.

15. J. W. Cronin et al., Phys. Rev. D 11 (1975) 3105. D. Antreasyan et al., Phys. Rev. D 19 (1979) 764;

16. W. K. Brooks, talk at Jefferson Laboratory Users Group Workshop, June 13, 2006.

17. E. Wang and X. N. Wang, Phys. Rev. Lett. 89 (2002) 162301.

18. F. Arleo, Eur. Phys. J. C 30 (2003) 213 and JHEP 0211 (2002) 044.

19. A. Bialas and M. Gyulassy, Nucl. Phys. B 291 (1987) 793.

20. A. Accardi, V. Muccifora and H. J. Pirner, Nucl. Phys. A 720, 131 (2003).

21. A. Accardi, D. Grunewald, V. Muccifora and H. J. Pirner, Nucl. Phys. A 761 (2005) 67

22. B. Z. Kopeliovich, J. Nemchik, E. Predazzi and A. Hayashigaki, Nucl. Phys. A 740 (2004) 211.

23. T. Falter, W. Cassing, K. Gallmeister and U. Mosel, Phys. Rev. C 70 (2004) 054609.

24. Y. L. Dokshitzer, V. A. Khoze, A. H. Mueller and S. I. Troian, "Basics of perturbative QCD,", Ed. Frontieres, Gif-sur-Yvette, France, 1991. 
25. R. Baier, Y. L. Dokshitzer, A. H. Mueller and D. Schiff, JHEP 0109, 033 (2001)

26. C. A. Salgado and U. A. Wiedemann, Phys. Rev. D 68 (2003) 014008.

27. A. Accardi, arXiv:nucl-th/0510090

28. B. Andersson et al., Phys. Rept. 97 (1983) 31.

29. X. N. Wang, Phys. Lett. B 579 (2004) 299

30. H. P. Blok and L. Lapikas, Phys. Rev. C 73 (2006) 038201

31. A. Accardi, arXiv:nucl-th/0604041

32. B. A. Kniehl, G. Kramer and B. Potter, Nucl. Phys. B 582 (2000) 514.

33. R. Baier et al., Nucl. Phys. B 484 (1997) 265;

34. M. B. Johnson et al., Phys. Rev. C 63 (2001) 035203.

35. S. S. Adler et al. [PHENIX Collaboration], Phys. Rev. Lett. 96 (2006) 202301.

36. A. Deshpande et al. Ann. Rev. Nucl. Part. Sci. 55 (2005) 165 